\title{
BEAM DYNAMICS STUDIES FOR THE FAULT TOLERANCE ASSESSMENT OF THE PDS-XADS LINAC DESIGN*
}

\author{
J-L. Biarrotte, CNRS/IN2P3/IPNO, Orsay, France \\ M. Novati, P. Pierini", INFN/LASA, Segrate (MI), Italy \\ H. Safa, D. Uriot, CEA/DSM/DAPNIA/SACM, Gif-sur-Yvette, France
}

\begin{abstract}
In order to meet the high availability/reliability required by the PDS-XADS design, the accelerator needs to implement to the maximum possible extent a fault tolerance strategy that would allow beam operation in the presence of most of the envisaged faults that could occur in its beamline components. In this work, we report the results of beam dynamics simulations performed to characterize the effects of the faults of the main linac components (cavities and focusing magnets) on the beam parameters. The outcome of this activity is the definition of the possible corrective actions that could be conceived (and implemented in the system) in order to guarantee the fault tolerance characteristics of the accelerator.
\end{abstract}

\section{INTRODUCTION}

In current high-power accelerators, when a component fails, the beam is immediately shut down, and action is then taken to repair the faulty component. After which, when the accelerator operation is ready to resume again, the procedure followed is quite identical to a new re-start, slowly ramping up in beam power. For most accelerators, the number of beam interruptions rapidly decreases with the trip duration time, thus maintaining an overall good level of availability. But for an ADS application, any beam trip lasting more than one second will be considered as a major accelerator failure, leading to the reactor core shutdown. Thus, the philosophy prevailing on current machines to cope with component failures should be reconsidered, taking into account this requirement. In particular, for each failure analysis, the design should look at the ability to either maintain the beam under safe conditions, or to recover the beam through, in less than one second. This is a new feature, not required for any other accelerator application, which is quite specific to ADS linacs. In this paper, we will develop this "fault tolerance" concept, and try to assess its practical implementation through beam dynamics calculations.

\section{THE PDS-XADS ACCELERATOR}

Consecutive to the work of the European Technical Working Group on Accelerator Driven Systems [1], the Preliminary Design Study of an eXperimental ADS (PDSXADS) was launched in 2001. A large European collaboration supported by the EU within the $5^{\text {th }}$ Framework Program (FP) performs these studies [2]. Five work packages (WP) cover the relevant issues; the WP3 is

\footnotetext{
* The PDS-XADS program is funded by the EC $5^{\text {th }}$ Framework Program, under contract FIKW-CT-2001-00179.

\#paolo.pierini@mi.infn.it
}

dedicated to the design of the high power proton accelerator (HPPA) providing the neutron flux to the subcritical reactor via a spallation target.

\section{The reliability requirement}

The XADS accelerator's main specifications are quite usual for such an HPPA machine: $600 \mathrm{MeV}$ final energy, $6 \mathrm{~mA} \mathrm{CW}$ maximum beam current (10 mA rated), 2\% beam power stability, $10 \%$ beam size stability on target.

On the other hand, less than a few (in the order of 5 per year) beam stops longer than approximately one second are allowed for the successful demonstration of the ADS coupling. Given the state-of-the-art in the field of accelerator reliability, this requirement appears to be highly challenging, and could reveal itself as being a "show-stopper" for ADS technology. From this extremely high reliability requirement, it is clear that suitable design strategies had to be followed early in the conception stage of the XADS accelerator. The main guidelines that have been highlighted to drive the design are a strong design (which makes extensive use of component derating and redundancy) and a high degree of fault tolerance (i.e. the capability to maintain beam operation within nominal conditions under a wide variety of component faults).

This fault-tolerance concept is a crucial point in the design of the XADS accelerator. The state of the art in RF system technology is indeed not reliable enough to envisage an operation of the XADS accelerator during several months without beam trips. We can actually foresee at least a few tens of failures per year, only due to these systems, based on parts count reliability estimates. Therefore, even if a great effort can be surely directed at improving the MTBF of RF systems, it seems difficult to reach the reliability requirements without implementing any fault-tolerance philosophy for the linac design.

\section{The XADS reference accelerator}

From this, WP3 has assessed the corresponding technical answers, and a reference solution based on a linear superconducting (SC) accelerator with its associated doubly achromatic beam line has been worked out up to some detail [3]. The injection section is composed of a "classical" proton injector (ECR source + normal conducting RFQ structure), followed by additional warm IH-DTL or/and superconducting CH-DTL up to a transition energy still to be defined (between 5 and $50 \mathrm{MeV})$. In this part, fault-tolerance is guaranteed by means of a "hot stand-by" spare. Above this transition energy, a fully modular SC linac brings the beam up to the final energy, using spoke and elliptical cavities. This section is designed to be intrinsically fault tolerant, 
meaning that an individual cavity failure can be handled at all stages without loss of the beam. This characteristic relies on the use of highly "de-rated" and independently powered accelerating components, associated to a fast digital feedback system and adequate diagnostics.

In the following, the fault-tolerance principle of this modular SC linac will be analysed by means of beam dynamics simulations. The calculations are performed using the TraceWin \& Partran codes developed in Saclay [4], and on the basis of a $5 \mathrm{MeV}-600 \mathrm{MeV}$ XADS reference linac (see Table 1). In all cases, a $10 \mathrm{~mA}$ proton beam is considered, and the normalized rms emittances values at the input are assumed to be $0.27 \pi . \mathrm{mm} . \mathrm{mrad}$ in the transverse planes, and $0.39 \pi \cdot \mathrm{mm} . \mathrm{mrad}$ in the longitudinal plane. Multiparticle calculations are performed using at least 10000 particules with a gaussian (truncated at $4 \sigma$ ) phase-space distribution.

Table 1: Lay-out of the linac used for the simulations; focusing is ensured by warm quadrupole doublets.

\begin{tabular}{|l|c|c|}
\hline SC linac sections & Energy range & Nb of cavities \\
\hline $\begin{array}{l}\text { Spoke } \text {-gap } \\
352.2 \mathrm{MHz}, \beta=0.15\end{array}$ & $5-17 \mathrm{MeV}$ & $\begin{array}{c}36 \\
\text { (2 per lattice) }\end{array}$ \\
\hline $\begin{array}{l}\text { Spoke } 2 \text {-gap } \\
352.2 \mathrm{MHz}, \beta=0.35\end{array}$ & $17-91 \mathrm{MeV}$ & $\begin{array}{c}63 \\
(3 \text { per lattice) }\end{array}$ \\
\hline $\begin{array}{l}\text { Elliptical 5-gap } \\
704.4 \mathrm{MHz}, \beta=0.47\end{array}$ & $91-192 \mathrm{MeV}$ & $\begin{array}{c}28 \\
(2 \text { per lattice })\end{array}$ \\
\hline $\begin{array}{l}\text { Elliptical 5-gap } \\
704.4 \mathrm{MHz}, \beta=0.65\end{array}$ & $192-498 \mathrm{MeV}$ & $\begin{array}{c}51 \\
(3 \text { per lattice })\end{array}$ \\
\hline $\begin{array}{l}\text { Elliptical 6-gap } \\
704.4 \mathrm{MHz}, \beta=0.85\end{array}$ & $498-615 \mathrm{MeV}$ & $\begin{array}{c}12 \\
(4 \text { per lattice) }\end{array}$ \\
\hline
\end{tabular}

\section{TOLERANCE TO A RF CAVITY FAULT}

\section{Consequences of the failure of a $R F$ cavity}

Let us assume in this section that the RF system fails to power a cavity somewhere in the superconducting linac, and that this cavity is immediately detuned to avoid the beam loading effect. This results in a loss of the energy gain provided by the failed cavity, and then in a beam longitudinal (phase-energy) mismatch at the entrance of the following cavity. Because we deal with a nonrelativistic proton beam, this energy loss will imply a phase slip along the linac equal to $\delta \phi \approx 2 \pi(\delta z / \lambda)\left(\delta \beta / \beta^{2}\right)$, increasing with the distance $\delta z$ from the faulty cavity [5]; $\beta$ is the beam velocity (normalized to $c$ ), $\lambda$ the RF wavelength and $\delta \beta$ the velocity loss (compared to the reference beam velocity) at $\delta z$.

Of course, the consequences of such a failure strongly depend on the position of the cavity in the linac, and on its operating conditions. The problem is more serious when the velocity of the particle is low, but also when the accelerating field and the operating frequency of the cavity are high. Another important parameter to be taken into account in the analysis is the longitudinal acceptance of the linac. The lower is this acceptance (i.e. the higher is the synchronous phase compared with the longitudinal size of the beam), the faster the fault-induced phase slip will off-set the beam towards the phase instability region. One way to avoid the problem could thus be to lower both the synchronous phase (near the $-90^{\circ}$ bunching value) and the accelerating fields along all the linac, but this would lead to an unacceptable increase in length and cost.

In our XADS reference linac, where conservative but realistic synchronous phase and accelerating gradient values are used, simulations show that the fault of a cavity induces a sufficient phase slip to rapidly drop the beam out of the phase stability region. The beam can then not be correctly handled longitudinally in the subsequent cavities, and it is finally completely lost later in the linac. This kind of behaviour with a final $100 \%$ beam loss is encountered for almost any cavity fault in the linac.

\section{Retuning using the local compensation method}

From this first analysis, it is clear that in case of a cavity failure, some kind of retuning has to be performed. The aim of this retuning is to recover the nominal beam characteristics at the end of the linac, and in particular its energy, while ensuring the same level of beam transmission (and of emittance growth) than in the reference linac case.

One simple way to achieve such a retuning is to make a local compensation by using the accelerating cavities neighbouring the failing one. This method has especially the advantage of involving a small number of elements, simplifying the retuning procedures and limiting the possible induced errors. It is illustrated on Figure 1: if cavity $\# \mathrm{n}$ is faulty, the 4 surrounding cavities (\#n-2, \#n-1, $\# \mathrm{n}+1, \# \mathrm{n}+2)$ are retuned to recover the nominal beam energy and phase at the end of the following lattice (point M). It can of course be done with more cavities if necessary. Practically, the retuning of the cavities is made only acting on their accelerating field amplitude and/or phase. On the transverse beam dynamics side, the gradients of the 4 focusing quadrupoles located inside the retuned lattices can also be adjustedm if needed. Here again, more quadrupoles can be used if necessary.

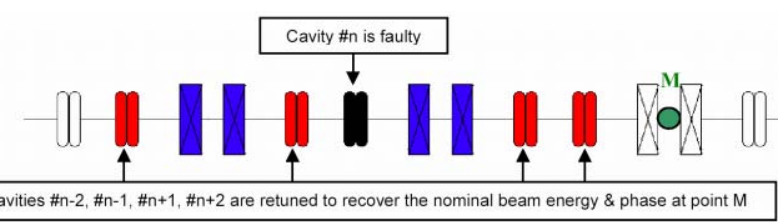

Figure 1: The "local compensation" method.

This retuning has of course to be done properly in order to reach a reasonable compromise between the three following goals: 1 . reach the nominal energy and phase at point $\mathrm{M}$, and consequently at the target; 2 . limit the induced beam mismatch to ensure a $100 \%$ transmission and keep the emittance growth as low as possible; 3 . ensure that we do not exceed a $+30 \%$ accelerating field increase in the cavities as compared with their nominal "derated" operation point (i.e. in our case $\mathrm{E}_{\mathrm{pk}}=33 \mathrm{MV} / \mathrm{m}$ instead of $25 \mathrm{MV} / \mathrm{m}$ in the spoke sections, and $\mathrm{B}_{\mathrm{pk}}=$ $65 \mathrm{mT}$ instead of $50 \mathrm{mT}$ in the elliptical sections). 
A reasonable compromise between these three requirements is not always easy to reach, especially in the very low velocity section (spoke $\beta=0.15$ ) where the beam is debunching very rapidly at the fault cavity position, leading to halo creation and beam losses if a refined retuning is not carried out. In this specific case, acceptable beam behaviour can be restored (see Figure 2) by performing a strong longitudinal focusing in the retuned cavities to compensate for this effect. Above $10 \mathrm{MeV}$, the situation becomes far easier, and the nominal beam specifications can be broadly restored.

A systematic study of the XADS linac fault-tolerance has been performed, optimizing the retuned values to be applied for local compensation in the case of the failure of most of the linac cavities. Detailed results can be found in [6]. The conclusion of the study is that in every case, with an appropriate retuning, the beam can be transported up to the high energy end without any beam loss $(100 \%$ transmission, reasonable emittance growth), and within the nominal target parameters.

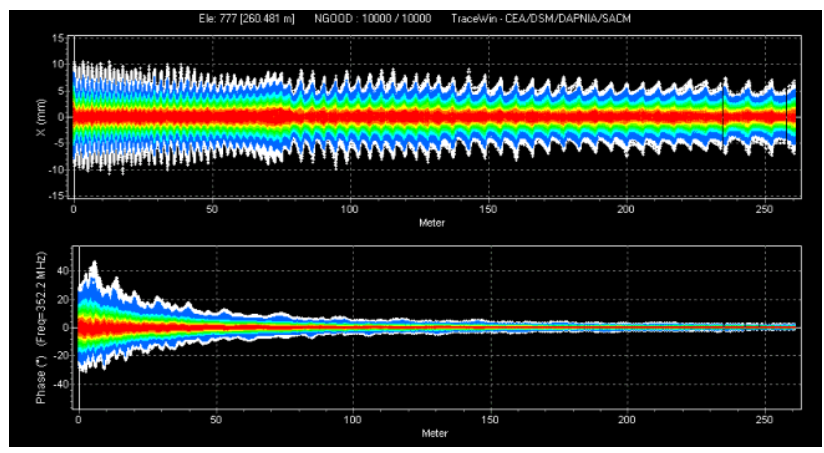

Figure 2: Multiparticle beam envelopes (x-transv., phase) in the XADS linac if spoke cavity \#4 $(5.5 \mathrm{MeV}, 3 \mathrm{~m})$ is faulty and local compensation is performed.

\section{CASE OF A QUADRUPOLE FAILURE}

Calculations have been also performed to analyse the case where a beam focusing quadrupole is failing. Here again, the consequences of such a failure depend on the position of the failed quadrupole. The situation is more critical in the sections where the safety ratio between the beam tube aperture and the beam size is smaller. For example, if no retuning at all is performed, the failure of one of the very first linac quadrupoles leads to a beam loss of about $30 \%$, while the same failure in the $\beta=0.35$ spoke section only leads to a beam loss of about $10 \%$. In the elliptical sections, the induced beam loss is always lower than $5 \%$.

Of course, if no retuning is done, the beam is strongly mismatched in the transverse planes from the failure position up to the linac end. But simulations clearly show that the situation can be substantially improved if the other quadrupole of the faulty doublet is also switched off. As a matter of fact, the induced mismatching is thus better balanced between the 2 transverse planes than if nothing is done. It is thus recommended to switch off the whole doublet if one quadrupole fails.
Finally, with an adequate retuning of the surrounding quadrupole doublets (local compensation method), it is generally possible to rematch the beam to the linac and obtain beam envelopes very similar to the nominal case ones. Detailed results can be found in [6].

\section{CONCLUSION}

The systematic analysis performed to evaluate the faulttolerance capability of the XADS superconducting linac leads to the following conclusions:

- If a cavity fails and if nothing is done, the beam is (almost) always completely lost.

- If a cavity fails and if an appropriate local compensation is done (retuning of a few surronding cavities field and phase + adjustement of a few quadrupoles gradients if needed), the nominal beam parameters at the target can always be restored.

- If a quadrupole fails, the whole doublet has to be switched off to limit the beam losses along the accelerator. The nominal beam parameters at the target can generally be restored by readjusting a few surrounding quadrupole gradients.

- The situation is substantially more difficult in the very low energy section of the linac $(<10 \mathrm{MeV})$.

This analysis is anyway not complete because it only takes into account the beam behavior before the failure, and after local compensation. The remaining part of the problem consists now in analysing what happens during the transient state, and in optimizing the way the retuning procedure is carried out in order to minimize the possible beam losses due to these transients. This full analysis will be performed within the $6^{\text {th }}$ FP project EUROTRANS, with the development of adequate simulation codes, and the construction of a real accelerating cryomodule, equipped with its associated RF control systems, to demonstrate the proof of principle of the technology [7].

\section{REFERENCES}

[1] “A European Roadmap for Developing Accelerator Driven Systems (ADS) for Nuclear Waste Incineration”, ISBN 88-8286-008-6, Rome, 2001.

[2] B. Carluec, "The European project PDS-XADS", Int. Wor. for P\&T and ADS, Mol, 2003.

[3] See, e.g., contributions of A. C. Mueller, P. Pierini \& H. Safa at the Int. Wor. for P\&T and ADS, Mol, 2003.

[4] R. Duperrier, N. Pichoff \& D. Uriot, "CEA Saclay codes review", ICSS 2002 conf., Amsterdam, 2002.

[5] N. Pichoff \& H. Safa, "Reliability of superconducting cavities in a high power proton linac", $7^{\text {th }}$ EPAC conference, Vienna, 2000.

[6] J-L. Biarrotte \& al., "Beam dynamics studies for the fault tolerance assessment of the PDS-XADS linac design", OECD/NEA $4^{\text {th }}$ Int. Wor. on Utilisation \& Reliability of HPPA, Daejeon, Korea, 2004.

[7] T. Junquera, "Status and perspectives of the R\&D programs for the XADS linear accelerator", Int. Wor. for P\&T and ADS, Mol, 2003. 